\title{
Linezolid induced delirium in the absence of serotonin syndrome: a psychiatric consultation/liaison case report
}

\author{
Hani Nazha MD ${ }^{1}$, Nathan T. Harrington $\mathrm{MD}^{2}$ \\ Author Affiliations: \\ 1. West Virginia University, Morgantown, West Virginia \\ 2. West Virginia University - CAMC, Morgantown, West Virginia
}

The authors have no financial disclosures to declare and no conflicts of interest to report.

\section{Corresponding Author:}

Hani Nazha MD

West Virginia University

Morgantown, West Virginia

Email: hanazha@hsc.wvu.edu 


\section{Abstract}

Delirium is one of the most common mental illnesses that can affect elderly patients and patients with advanced medical problems. Because these patients are frequently on multiple medications and/or are more sensitive to medications secondary to their age, interactions with current medications, or existing medical problems, medication toxicity is frequently the etiology behind their delirium. This is a case report of a patient admitted for cellulitis that developed delirium from Linezolid however did not develop any other signs or symptoms of serotonin syndrome; a known side effect of Linezolid. This distinctive case highlights the importance of a careful analysis of a patient's medications for potential deliriogenicity as well as the value of using a validated tool for assessing and following up a patient with suspected delirium. Specifically, this case should give clinicians warrant for suspicion of Linezolid as the cause of a patient's delirium regardless of the presence or absence of serotonin syndrome.

\section{Keywords}

Linezolid, serotonin syndrome, delirium, DRS

\section{Introduction}

Nearly 30 percent of all older medical patients experience delirium at some point during their hospitalization and this is one of the most common mental illnesses to develop in the elderly and in patients with advanced medical problems. ${ }^{1}$ Early identification that patients are in a delirious state and the prompt treatment of the medical etiology or stopping of the deliriogenic agent are crucial in decreasing morbidity and mortality in these patients. An important first step in the evaluation of a delirious patient is a thorough review of the patient's medication list for medications known to cause delirium (see Table 1) as approximately 30 percent of cases are drug-induced. ${ }^{2}$ Additionally, the use of a validated and standardized assessment tool can be valuable in identifying and monitoring a delirious patient. ${ }^{3}$ Lastly, formulation of a comprehensive working differential diagnosis is critical in managing these patients. 
Table 1. Potential Deliriogenic Medications

\begin{tabular}{|l|l|}
\hline Analgesics & Antidepressants \\
\hline NSAIDs & Mirtazapine \\
\hline Opioids-specifically meperidine & SSRI's \\
\hline Antibiotics & TCA's \\
\hline Acyclovir & Antiarrhythmics \\
\hline Aminoglycosides & Beta blockers \\
\hline Amphotericin B & Clonidine \\
\hline Antimalarial drugs & Digoxin \\
\hline Cephalosporins & Diuretics \\
\hline Cycloserine & Methyldopa \\
\hline Fluoroquinolones & Corticosteroids \\
\hline Isoniazid & Dopamine agonists \\
\hline Interferon & Amantadine \\
\hline Linezolid & Bromocriptine \\
\hline Macrolides & Levodopa \\
\hline Metronidazole & Pergolide \\
\hline Nalidixic acid & Pramipexole \\
\hline Penicillins & Ropinirole \\
\hline Rifampin & Gastrointestinal agents \\
\hline Sulfonamides & Antiemetics \\
\hline Anticholinergics & Antispasmodics \\
\hline Atropine & Histamine-2 receptor blockers \\
\hline Benztropine & Loperamide \\
\hline Diphenhydramine & Herbal supplements \\
\hline Scopolamine & Belladonna extract \\
\hline Trihexyphenidyl & Henbane \\
\hline Anticonvulsants & Mandrake \\
\hline Carbamazepine & Jimson weed \\
\hline levetiracetam & St. John's Wort \\
\hline Phenytoin & Valerian \\
\hline Valproate & Phenothiazines \\
\hline Vigabatrin & *not a complete list, all medication should be \\
considered. \\
\hline & \\
\hline
\end{tabular}


This case report focuses on Linezolid, an oxazolidinone antibiotic that also has reversible monoamine oxidase (MAO) inhibition properties. It is well known that when used concomitantly with other serotonergic medications, Linezolid increases the patient's susceptibility towards developing serotonin syndrome. Also, inhibition of mammalian mitochondrial protein synthesis by oxazolidinones including linezolid has been studied and reported; this is believed to be secondary to oxidative injury from prolonged use.

While it has been theorized that the increase in catecholamines from monoamine oxidase inhibition could also be deliriogenic, this case is unique in that similar cases have not been commonly documented in the literature where Linezolid is the cause of delirium in the complete absence of serotonin syndrome.

\section{CASE REPORT}

A morbidly obese (BMI of 45) Caucasian female in her mid-fifties who smokes about two packs per day presented to her local emergency room with complaints of persistent moderate left lower extremity pain secondary to cellulitis that had been associated with nausea, vomiting, and fever for the past three days. She has had recurrent cellulitis and MRSA infections along with a medical history significant for poorly controlled DM2, CKD stage III, anemia of chronic disease, COPD, and hypertension. Patient does not have past medical history of any psychiatric illnesses and takes no psychotropic medications.

She was appropriately admitted from the emergency room to medicine service and was started on Vancomycin and Piperacillin/Tazobactam and given IV fluids; patient's lactic acid was mildly elevated upon admission $(2.3 \mathrm{mmol} / \mathrm{L})$ but normalized with the use of normal saline. Her cellulitis was demarcated to track improvement and MRI and venous duplex scans were performed to rule out osteomyelitis, abscess, and deep venous thrombosis; all were negative.

After several days the patient began to improve clinically as her vitals returned to normal, WBC's trended down, and the erythema receded to approximately the mid-calf. It was at this time in her hospital course that she developed an acute kidney injury. Nephrology thought this was likely due to intrinsic renal disease and recommended stopping Vancomycin and Piperacillin/Tazobactam. Her acute kidney injury resolved and an infectious disease consultation recommended starting Linezolid. The patient was started on Linezolid 600mg IV, given two doses, and over the next 24 hours developed an altered mental status. Psychiatry consultation/liaison service was consulted to evaluate the patient at that time.

Psychiatry evaluation and assessment included the Delirium Rating Scale (DRS-R-98) which was used to evaluate and follow up the patient. The patient scored $30 / 39$ on the severity scale and 33/46 total score. Clinically she had day-night reversal, was completely disoriented and inattentive, was having auditory and visual hallucinations, exhibited a lability of affect with hysterical laughing, crying, and yelling; she required a 24 hour sitter and needed assisted feeds. The teams' differential diagnosis included serotonin syndrome secondary to the newly started Linezolid or a delirium secondary to sepsis, uremic encephalopathy, or medications.

Serotonin syndrome was ruled out by using The Hunter Serotonin Toxicity Criteria ${ }^{4}$. While the patient had been administered Linezolid, a serotoninergic medication, she was not on any other 
serotonergic medications, and was afebrile and without tremor, rigidity, or clonus. Sepsis was ruled out as the cause of her delirium as her sepsis was resolving when she became delirious; her WBC's had been trending down, and her vital signs had been stable for more than 24 hours. Uremia was not likely the cause of her delirium as her BUN was never more than 60, and by the time she was switched to Linezolid her creatinine levels had returned to baseline. The patient was not on any other medications likely to be deliriogenic and it was determined that it was likely the Linezolid causing the patient's delirium despite the absence of any serotonin syndrome symptoms. Linezolid was stopped in the initial evaluation by the consult team and within 24 hours of discontinuation the patient's mental status had returned to her baseline. On the Delirium Rating Scale the patient now scored 6 on the severity scale and 7 on the total scale, and this did not fluctuate for the rest of her hospital stay. (See Figure 1)

Figure 1

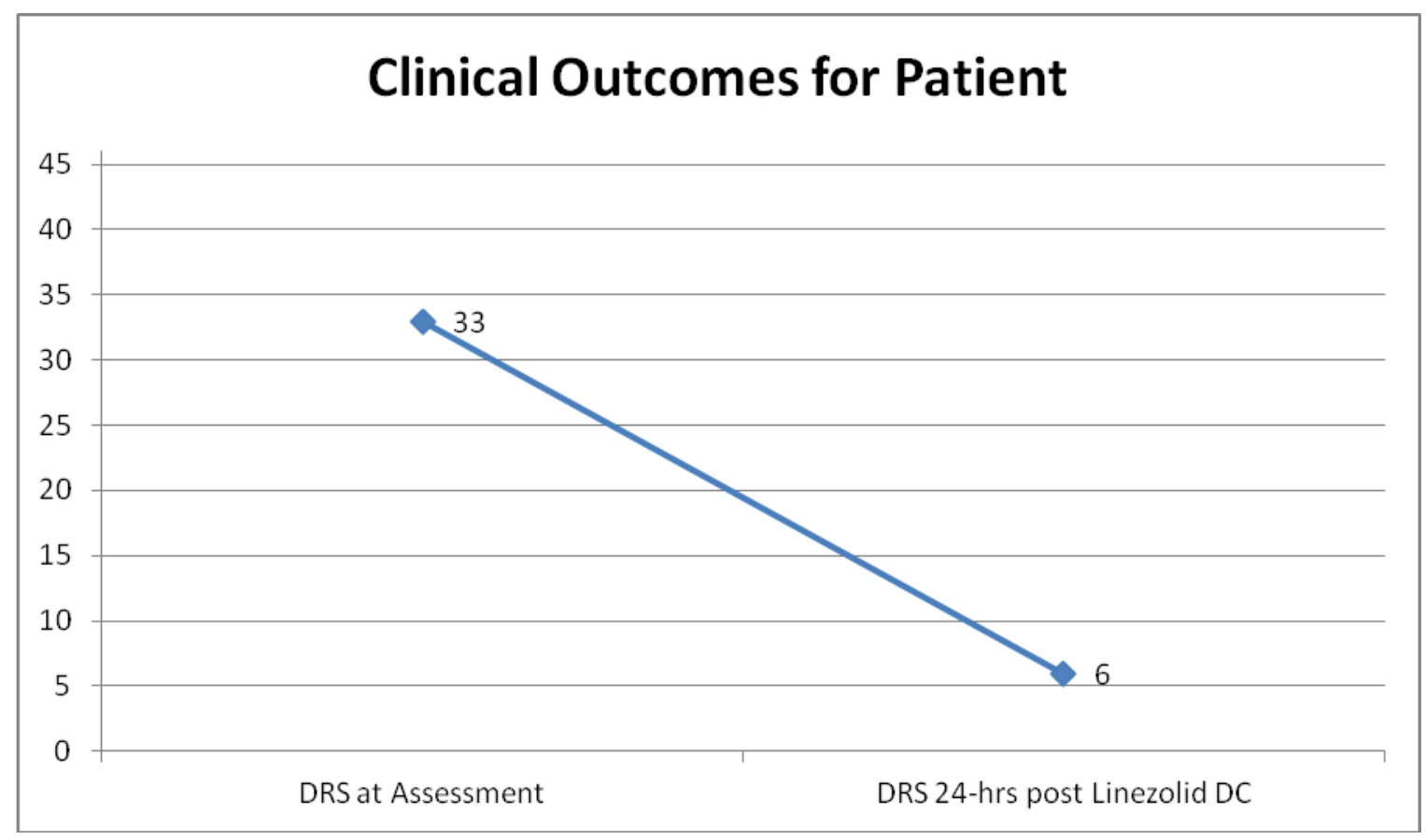

\section{DISCUSSION}

This unique case demonstrates the two key clinical aspects of care in a patient with delirium: the use of a standardized assessment tool to identify and follow up a patient with delirium and the formulation of a comprehensive differential diagnosis that includes a thorough analysis of the patient's medications. The use of a validated assessment tool for delirium, such as the DRS-R98, can help give a much clearer clinical picture of the delirious patient's fluctuations and response to treatment. ${ }^{3}$ Indeed, the Delirium Rating Scale R-98 was valuable in determining the effect of our intervention with this patient and confirming the suspected etiology; this scale can be administered by physicians or nurses. Although the pathophysiology of delirium is poorly 
understood, theoretically any medication that can cause a disruption in the normal neurotransmitter pathways can precipitate a delirium. Delirium is essentially the final common symptom of multiple neurotransmitter abnormalities including the acetylcholine, serotonin, glutamate, GABA, and dopamine neural pathways leading to increased glutamate, decreased GABA, and increased dopamine. ${ }^{5}$ Linezolid acts to inhibit both MAO-A and MAO-B reversibly. MAO-A inhibition primarily increases available serotonin, whereas MAO-B inhibition primarily increases available dopamine and norepinephrine. It is this separate MAO-A and MAO-B binding of Linezolid that may be why our patient developed her delirium without serotonin syndrome. Clinicians should be mindful that in patients treated with Linezolid, who subsequently develop altered mental status, the discontinuation of Linezolid should be considered regardless of the presence or absence of serotonin syndrome.

\section{REFERENCES}

1. Francis J. Delirium in older patients. J Am Geriatr Soc. 1992;40(8):829.

2. Francis J. Drug-induced delirium: Diagnosis and treatment. CNS Drugs. 1996;5:103.

3. Trzepacz $\mathrm{P}$ et al. Validation of the delirium rating scale-revised-98: comparison with the delirium rating scale and the cognitive test for delirium. J Neuropsychiatry Clin Neurosci. 2001;13:229-242

4. Dunkley EJC et al. The Hunter Serotonin Toxicity Criteria: simple and accurate diagnostic decision rules for serotonin toxicity. QJM. 2003;96 (9)635-642.

5. Flacker JM, Lipsitz LA. Neural mechanisms of delirium: current hypotheses and evolving concepts. J. Gerontol. A Biol. Sci. 1999;54(7):B275.

6. Serio RN. Acute delirium associated with combined diphenhydramine and linezolid use. Annal Pharmacotherapy. 2004;38(1):62-5.

7. Mattappalil A, Mergenhagen K. Neurotoxicity with antimicrobials in the elderly: a review. Clin Ther. 2014; 36(11):1489-1511. 